\title{
Passive Balancing of the Rotor with an Auto-Balancing Device with a Viscous Incompressible Liquid
}

\author{
Ilona DRACH*, Vilen ROYZMAN**, Algimantas BUBULIS***, Kazimieras JUZENAS**** \\ *Khmelnitsky National University, Instutytska 1, 29016 Khmelnitsky, Ukraine, E-mail: cogitare410@gmail.com \\ **Khmelnitsky National University, Instutytska 11, 29016 Khmelnitsky, Ukraine \\ ****Kaunas University of Technology, Studentu 56, LT-51424 Kaunas, Lithuania, E-mail: algimantas.bubulis@ktu.lt \\ ****Kaunas University of Technology, Studentu 56,LT-51424 Kaunas, Lithuania, E-mail: kazimieras.juzenas@ktu.lt \\ crossref http://dx.doi.org/10.5755/j02.mech.23789
}

\section{Introduction}

Modern methods and means of balancing allow us to bring the initial imbalance of a manufactured or repaired rotor to a value acceptable for a given class of balancing precision. However, there may be a change of the initial imbalance, which is the result of a technological operation mode while operating the machine. The change of imbalance occurs at different rates of operation and usually has a random nature.

To compensate for the change of the imbalance, additional balancing in the machine-off state may be carried out. Such balancing involves stopping the machine and has high economic costs. In some cases, the stop is undesirable due to requirements of the technological process or is unacceptable at certain operating conditions. Therefore, the most promising, and often the only possible, is application of the auto-balancing devices (SBDs), which automatically compensate the change of rotor imbalance under operating conditions without stopping a machine.

The first simple example of a passive SBD has been developed more than 120 years ago. Subsequently, different types of such devices, including liquid ones, were developed. The correction mass (the liquid) not only creates an effort to balance the rotor, but also is a sensory element that responds to a change of imbalance. However, the analysis of the current state of the theory and practice of automatic balancing allows us to conclude that the existing theory of fluid SBD is limited to the consideration of an idealized rotor system without damping and without taking into account the hydrodynamic properties of the liquid. Liquid SBD are the most unexplored. There are no design methodologies that would take into account properties of liquids (density, viscosity, temperature, etc.). The practice of using liquid SBD showed that the theoretical conclusions, obtained when considering the idealized system, are not confirmed [1] in the real systems. This demanded an analysis of the operation of the liquid SBD in real systems taking into account the effect of external damping, which was done in [2]. The purpose of this article is to analyse the effect of liquid viscosity on the efficiency of the vertical rotor balancing process. The study of the dependence of the balancing efficiency on the viscosity of the liquid in the liquid SBD gives an opportunity to expand the areas of their application.

The history of liquid automatic balancing begins in 1916, when the French engineer Leblanc proposed a liquid SBD design to balance the extractor of the washing machine during its operation. Subsequently, Thearle's SBD and Dun- can's SBD and other enhancements to the designs of the Leblanc's auto-balancing devices were offered. Unfortunately, instructions on the use of these patented designs in real machines, were not found as well as information about their introduction into real products [3]. E. L. Thearle attempted to substantiate theoretically the principle of operation of Leblanc's SBD, ring, swivel, and ball SBDs in the cycles of his articles [4]. He proposed a flat model of the rotor and the auto-balancing device. Within the proposed framework, there is a single critical speed of the rotor, the excess of which causes the rotor to rotate the light side outward and to exhibit self-centred rotor phenomenon. Thearle described that as the fundamental for the operation of all passive SBDs. In fact, the phenomenon of self-centeredness appears as a phenomenon of self-balancing in this research.

The approaches and the results of E. L. Thearle's work formed the basis of the following studies: J. Larry, O. Gusarov [5], V. P. Nesterenko [6], H. S. Hoon, L. J. Young, S. Suzuki [7], Ye. M. Pashkov [8, 9], C. H. Jung, C. S. Kim, Y. H. Choi [10], M. A. Langthjem, T. Nakamura [11], M. W. M. Cunico, [12] H. W. Chen, W. X. She, Q. J. Zhang, Y. Cao and S. Y. Fan [13] and other researchers working in the field of machines rotors balancing with passive liquid SBD. Main results are obtained without taking into account the specific properties of correcting masses (liquid), the forces of resistance. Thus, those results are theoretically limited and experimentally unconfirmed. The performance of such automated balancing devices with the supercritical (after resonance) rotor rotation is still unexplored.

Such well-known companies as LG Electronics Inc.[14, 15], Whirlpool Corporation [16], SKF AutoBalancer Systems [17], Samsung Electronics Co. Ltd [18] are the main manufacturers and industrial users of passive SBDs (in particular, ball and liquid devices). However, information on the practical implementation of the abovementioned devices for automatic balancing of rotors of machines was not found. Such developments exist only as patented ideas. Companies use the idea of passive auto-balancing in advertising their products, which indicates the relevance of such SBDs. However, their widespread use in commercial products is hampered by many unresolved problems in the theory and practice of balancing rotors with passive SBD and the presence of a number of phenomena and contradictions that have not yet found the proper theoretical justification.

The presented research was triggered by the lack of studies on the effect of viscosity and density of the working fluid as well as on the efficiency of self-balancing and the 
validity of the selection of the working liquid in the development of fluid auto-balancing devices. Therefore, this research is aimed to analyse the performance of an experimental SBD, taking into account the influence of liquid properties on the efficiency of the vertical rotor balancing process.

\section{Results of theoretical research}

\subsection{The case of perfect liquid}

Let the vertical flexible rotor rotating on two rigid pivots at an angular velocity $\omega$ contains an SBD that has the form of a cylindrical cavity of radius $R$ and height $h$ with a liquid (an imbalance which is less than the rotor imbalance). Consider the mathematical model of fluid movement in the SBD chamber under the influence of a change in the total imbalance of the system and the deflection.

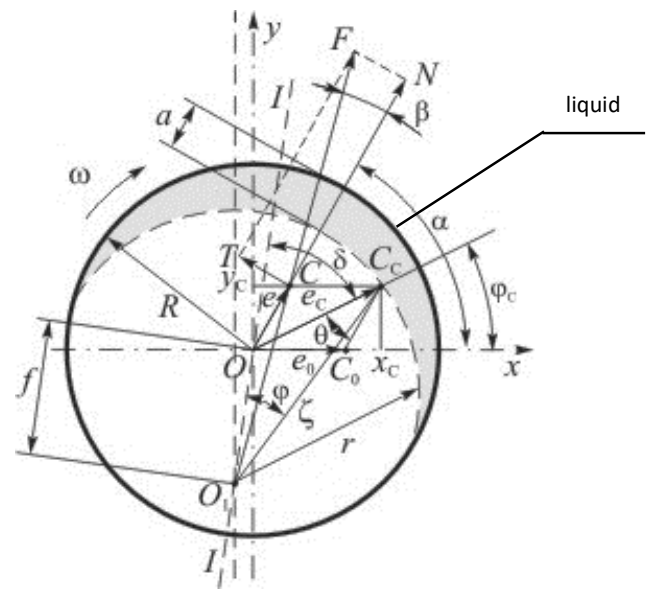

a

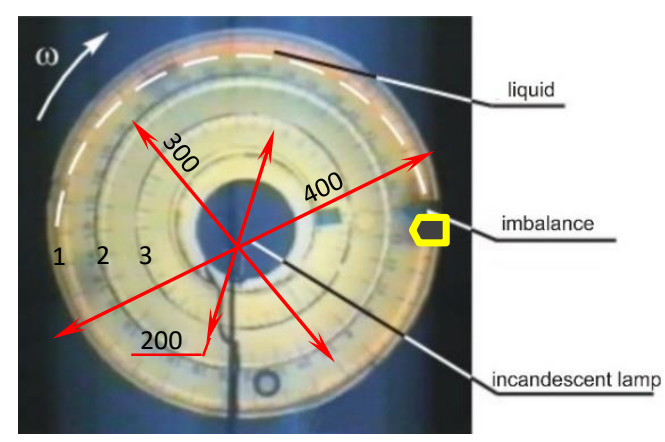

b

Fig. 1 Experimental auto-balancing device: a - scheme of forces acting in the SBD, $b$ - video frame of the experimental stand: $1,2,3$ - chambers of the device

Total imbalance of the rotor system - liquid $\overline{D_{c}}$ consists of a vector of the shaft imbalance $\overline{D_{0}}$ and fluid imbalance $\bar{D}$ let $O$ is a point of intersection of the axis of the curved shaft with the plane of the cavity (Fig. 1 a). $O_{1}-O_{1}$ is a bearing line; $C_{0}$ is centre of mass of the shaft; $C$ is centre of mass of fluid; $C_{c}$ is centre of mass of system shaft-liquid. $M$ is mass of the shaft; $m$ is mass of working fluid. $\overline{O C}=\bar{e}=\frac{\bar{D}}{(m+M)}$ is an "oriented" eccentricity of the cen- tre of the mass of the liquid; vector $\overline{O C_{0}}=\overline{e_{0}}=\frac{\overline{D_{0}}}{(m+M)}$ is an "oriented" eccentricity of the rotor mass $M$ and $\overline{O C_{c}}=\overline{e_{c}}=\frac{\overline{D_{c}}}{(M+m)}$ is the total ,oriented" eccentricity of the rotor with a liquid.

For the sake of clarity, theoretical calculations of the liquid positions in the SBD are checked on computer video frames (Fig. 1, b), obtained using the method of highspeed video shooting, described in detail in [1]. In order to study fluid behaviour in a balancing device in the pre-resonant and after-resonant zones of rotation of the rotor, an SBD model with optically transparent material has been designed and manufactured. The transparent setup allows visual tracing of SBD work in all modes of operation. The SBD is composed of an outer $\varnothing 400 \mathrm{~mm}$ diameter ring and two inner concentric partitions (diameters of Ø300 and Ø200 $\mathrm{mm}$ ), forming three concentric chambers for positioning of working bodies (Fig. 1, b). All three chambers are hermetically sealed from each other and may be separately filled with a working fluid. The imbalance mass of different size may be fixed in various positions.

At system speed $\omega<\omega_{c r}$ external resistance (forces of friction in bearings, forces of friction of a cylinder against air, etc.) causes a backlog of a deflection plane $(I-I)$ from the plane of total imbalance $\left(O C_{c}\right.$ ) on the corner $\delta$ (Fig. 1, a).

The liquid tends to take position in the most remote location from the axis of rotation. This leads to a change in the total imbalance of the system in terms of direction and magnitude. Since the magnitude of the total imbalance $\overline{D_{c}}=(M+m) \cdot \overline{e_{c}}$ is defined as the geometric sum of vectors $\overline{D_{0}}=M \cdot \overline{e_{0}}$ (rotor imbalance) and $\bar{D}=m \cdot \bar{e}$ (imbalance of the liquid) and depends on the angle between these vectors $\alpha$ (Fig. 1, a), which characterizes the position of the fluid relative to the rotor imbalance. The angle of lag (the deflection of the total imbalance) $\delta$ is constant for a certain angular velocity. The position of the deflection changes with respect to the initial imbalance $\overline{D_{0}}$ with the change in the direction of the total imbalance (that is, the angle of this lag with respect to the initial imbalance increases). The fluid following the deflection changes the total imbalance by increasing the angle $\alpha$ and reducing the size of the total imbalance. This leads to a decrease in the value of the deflection and changes its position relative to $\overline{D_{0}}$ (increase the angle of lag). Theoretically, this process is repeated until the angle of lag $\alpha$ will not reach the value of $180^{\circ}$, and the deflection will not reach the minimum value (or zero at the total equilibrium).

The total eccentricity of the rotor with a liquid will be determined as:

$$
e_{c}=\sqrt{x_{c}^{2}+y_{c}^{2}}=e \sqrt{1+2 k \cos \alpha+k^{2}},
$$

where: $k k=D_{0} / D$ is the ratio of rotor and fluid imbalances (see [19] for detailed description).

The efficiency of the balancing is characterized by the ratio of deviations from the axis of rotation of the centre 
of mass of the system without liquid and with the liquid $\lambda_{0}$ :

$$
\lambda_{0}=\frac{k}{\sqrt{1+2 k \cos \alpha_{0}+k^{2}}} .
$$

Balancing will be complete in the case if $1+2 k \cos \alpha_{0}+k^{2}=0$. Therefore the only solution will be $k=1$ with $\cos \alpha_{0}=-1$. Consequently, the greatest balancing effect is achieved when the major imbalance of the liquid is involved in the balancing process and is close to the initial value of the rotor imbalance $(k \approx 1)$. The liquid, which does not participate in balancing, is located in concentric circles, without affecting the total imbalance of the system.

\subsection{Case of viscous liquid}

In contrast to the ideal liquids, in real liquids, when moving some liquid layers, forces acting on the plane of contact between the layers are different. These forces are viscous friction (internal friction) or viscosity. Imaginary liquid may be divided into an infinite number of layers. Along the walls of the chamber, a thin layer of balancing liquid adheres to the surface and forms an adjoining layer. Its speed is equal to the speed of the system. Within this layer, the viscosity forces are as substantial as the forces of inertia. Outside of the adjacent layer, the velocity of the liquid layers decreases and the viscosity influence here decreases accordingly. In case of relative displacement on each of the layers, there are internal forces. From the side of the layer, whose velocity is greater, a force acts on the layer, whose velocity is lower. The direction of this force coincides with the direction of motion of the fluid. From the side of the layer whose velocity is smaller, a force that moves in the opposite direction from the motion of the fluid acts on a layer that moves at a higher speed, that is, it slows this layer. The force of internal friction in a liquid is proportional to the change in velocity in fluid layers and the coefficient of proportionality depends on the nature and state of the fluid [20]. When the liquid is in relative rest, this force turns to zero.

The amount of energy loss consumed by the forces of resistance is equal to [20]:

$$
\Delta P=\frac{4 F_{T}}{\pi d^{2}}
$$

where: $d$ is diameter of chamber section. Therefore:

$$
F_{T}=\frac{\pi}{4} \Delta P \cdot d^{2} .
$$

By the formula of Darcy [20] we have:

$$
\Delta P=\rho \cdot g \cdot \lambda \frac{l}{d} \frac{\mathrm{v}^{2}}{2 g}=\rho \lambda \frac{l}{d} \frac{\mathrm{v}^{2}}{2},
$$

where: $l$ is length of chamber; $v$ is average flow rate.

At low speeds (with $R e<R e_{c r}$ ) The friction force is mainly related with the viscosity of the fluid and the coefficient of friction resistance $\lambda=\frac{64}{R e}$ which follows from the Hagen- Poiseuille law [20]. Once $R e=\frac{v d}{n}=\frac{v r d}{m}$ (where $v$ is kinematic viscosity of the liquid, $\mu$ is dynamic viscosity of the liquid), then $\lambda=\frac{64 \cdot \mu}{\mathrm{v} \rho \cdot d}$. Therefore,

$$
\begin{aligned}
& F_{T}=\frac{\pi}{4} d^{2} \cdot \rho \lambda \frac{l}{d} \frac{\mathrm{v}^{2}}{2}=\frac{\pi d^{2} \rho \cdot 64 \mu \cdot l \cdot \mathrm{v}^{2}}{8 d^{2} \cdot \mathrm{v} \rho}=8 \pi \mu \cdot l \cdot \mathrm{v}=8 \pi \mu \cdot \frac{\pi R \xi}{180} \cdot \omega \cdot\left|\overline{O_{1} C}\right|= \\
& =\frac{8 \pi^{2} \mu \cdot R \xi \cdot \omega}{180} \sqrt{\left(e \cos \alpha+f \cos \left(\delta+\phi_{c}\right)\right)^{2}+\left(e \sin \alpha+f \sin \left(\delta+\phi_{c}\right)\right)^{2}},
\end{aligned}
$$

where: $\xi$ is the central angle defining the length of the arc of the motion of the fluid. In this case, the friction force is proportional to the speed of the camera's rotation and the viscosity of the liquid.

Friction in real systems leads to the fact that the liquid may stop in the not equilibrium position, but at some distance from it. Consequently, the liquid has a zone of equilibrium. The boundaries of the equilibrium zones are determined from the equilibrium condition of the fluid friction $F_{T}$ and the tangential component $T$ of the centrifugal forces of fluid inertia: $F_{T}=T$. Taking into account that $T=F \sin \beta$, we get that $\sin \beta=\frac{F_{T}}{F}$ or:

$$
\sin \beta=\frac{8 \pi^{2} \mu \cdot R \cdot \xi \cdot \omega\left|\overline{O_{1} C}\right|}{180 \cdot m \cdot \omega^{2}\left|\overline{O_{1} C}\right|}=\frac{8 \pi^{2} \mu \cdot R \cdot \xi}{180 \cdot m \cdot \omega} .
$$

Applying the trigonometric dependence $\operatorname{tg} \beta=\frac{\sin \beta}{ \pm \sqrt{1-\sin ^{2} \beta}}$ the equilibrium zones are determined by inequalities:

$$
-\frac{\frac{8 \pi^{2} \mu \cdot R \cdot \xi}{180 \cdot m \cdot \omega}}{\sqrt{1-\left(\frac{8 \pi^{2} \mu \cdot R \cdot \xi}{180 \cdot m \cdot \omega}\right)^{2}}} \leq \operatorname{tg} \beta \leq \frac{\frac{8 \pi^{2} \mu \cdot R \cdot \xi}{180 \cdot m \cdot \omega}}{\sqrt{1-\left(\frac{8 \pi^{2} \mu \cdot R \cdot \xi}{180 \cdot m \cdot \omega}\right)^{2}}} .
$$

Inequalities contain the angular velocity of the rotor $\omega$. Therefore, the boundaries of regions of the position of equilibrium of the viscous fluid depend on the angular velocity. Increase of angular velocity leads to a narrowing of the boundaries of equilibrium zones, i.e. the viscous fluid is located exactly against the imbalance. As the rotor's imbalance decreases, the role of the inertia forces acting on the liquid decreases and the role of frictional forces increases. 
These inequalities contain the coefficient of dynamic viscosity of liquid $\mu$. Boundaries of regions of the position of equilibrium of the viscous fluid depend on its internal friction. When the viscosity increases, the boundaries of the equilibrium zones expand.

Internal friction between layers of a real liquid prevents its movement. With a decrease in angle $\beta$, the tangential component of centrifugal force decreases and at some point cannot overcome the friction. Therefore, the balancing will be incomplete in the presence of viscosity. Results of the experimental studies confirm this stamen.

\section{Experimental research of SBD}

The installation for studying the behaviour of working bodies in the SBD has a rigid cantilever vertical rotor elastically suspended on the housing structure (Fig. 2). In order to provide sufficient rigidity of the rotor and the SBD mounted on it, the rotor is made of a stainless steel hollow cylinder with a bottom at one end, which is rigidly fixed on the shaft.

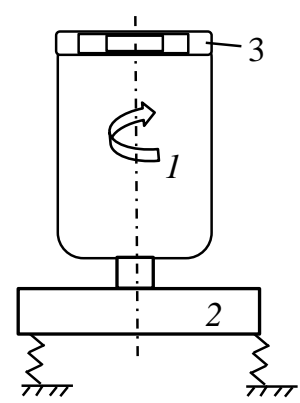

Fig. 2 Scheme of the experimental setup: 1 - hollow rotor, 2 - motor and belt transmission unit, 3 -experimental auto-balancing device

The shaft the rotor is associated through the bearings with rigid platform connected to the housing structure though four elastic suspension dampers. The natural frequency of the suspended system is approximately $9-10 \mathrm{~Hz}$. SBD is set and fixed by the outer diameter at the free end of the hollow cylinder (rotor). The necessary stiffness of fastening and positioning of the SBD on the rotor are assured. SBD layout has an internal hole sufficient for the free access to the inner cavity of the rotor, allowing change of imbalance location and size without dismantling the rotor. That is done by attaching a given mass to the inner wall SBD that is also held in its position by centrifugal forces.

The rotor is actuated by a synchronous electric motor ( $180 \mathrm{~W}$ power on a shaft) through the lowering transmission. Voltage of the motor is can be controlled in a wide range with the help of an autotransformer and control system. Therefore, the installation allows setting different frequencies of the rotor rotation in the range of $0.5-18 \mathrm{~Hz}(30-$ $1080 \mathrm{rpm})$ and control of angular accelerations when accelerating the rotor.

Investigations of the oscillations of the installation were carried out using a developed measuring system. The block scheme of the measuring equipment, united into a single vibration meter, is shown in the Fig. 3.

To record the amplitude-frequency characteristics (AFC) of the system, simultaneous recording of signals of all faze-adjusted sensors during the passage from zero to maximum speeds and with various values of the rotor's imbalance at these operating speeds was performed simultaneously. Signals of the rotor speed sensor were recorded in the same file with signals of the vibration sensors.

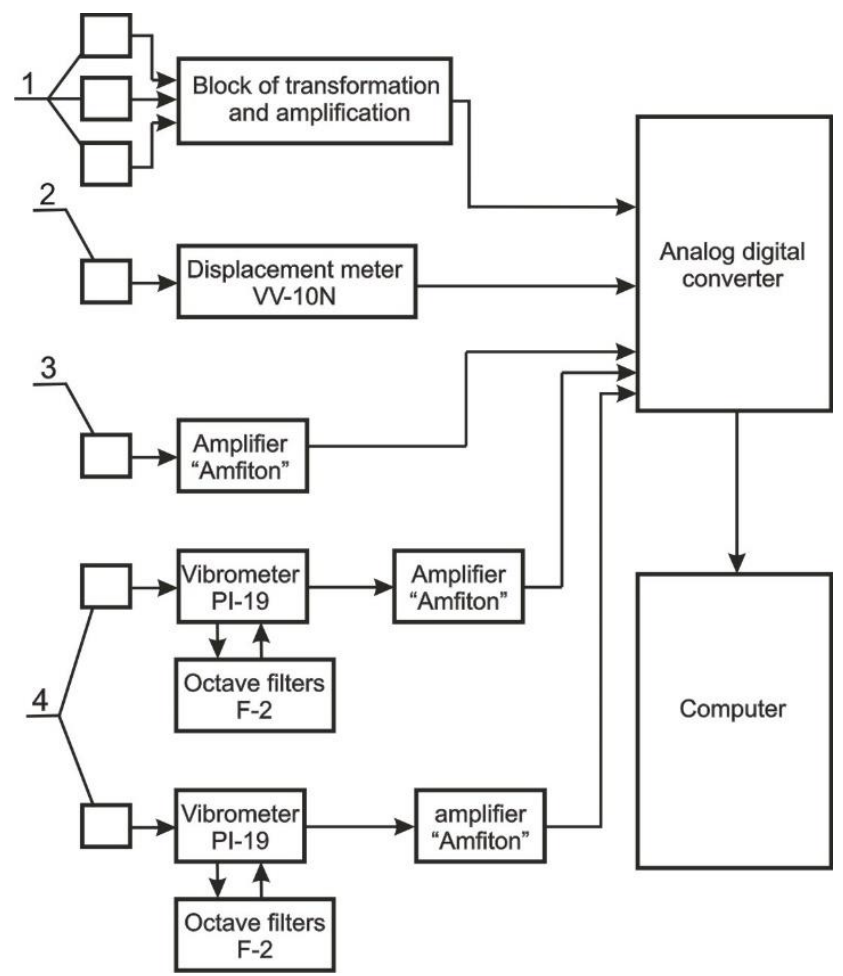

Fig. 3 Block scheme of equipment for the study of oscillations: 1 -photodetectors, 2 -inductive sensor, 3 - speed sensor, 4 - accelerometers

Record of sensors signals during the passage allows obtaining real amplitude-frequency characteristic of the studied system and evaluating influence of various factors, for example, starting torsion moment of the driving electric motor.

In order to exclude the effect of the mounting imbalance of the rotor itself on the results obtained, rotors balancing was carried out using method of bypassing loads.

The influence of liquid in the chamber density on the amplitude of oscillations and, accordingly, on the efficiency of balancing with different values of rotor imbalance was experimentally tested. Amplitude-frequency characteristics of the system oscillations at imbalances of $1000 \mathrm{~g}-\mathrm{cm}$ and $2000 \mathrm{~g}-\mathrm{cm}$ were recorded. Different working (balancing) liquids were selected: gasoline (refinin of benzene reformine TU-38, 5901471; density $670 \mathrm{~kg} / \mathrm{m}^{3}$ ), fresh water with a density of $1000 \mathrm{~kg} / \mathrm{m}^{3}$ and orthophosphoric acid with a density of $1650 \mathrm{~kg} / \mathrm{m}^{3}$. The balancing liquid was gradually poured into the chamber of $400 \mathrm{~mm}$ diameter with portions of $50 \mathrm{ml}$ (50-400 ml) without changing location and mass of the imbalance. After each change in the volume of the liquid, vibrations of the rotor were recorded at its passage and AFCs were composed.

Fig. 4 presents the dependences of the system oscillation amplitude on the volume of liquids in the SBD chamber. Results are obtained from AFCs in case of $2000 \mathrm{~g}-\mathrm{cm}$ imbalance, the system resonance frequency of $10 \mathrm{~Hz}$ and a shafts rotation frequency of $14 \mathrm{~Hz}$.

All curves (Fig. 4) represent the amplitudes of the vibrations of the upper edge of the unbalanced cylinder at 
constant mass and the position of the imbalance and the different volumes of working fluids.

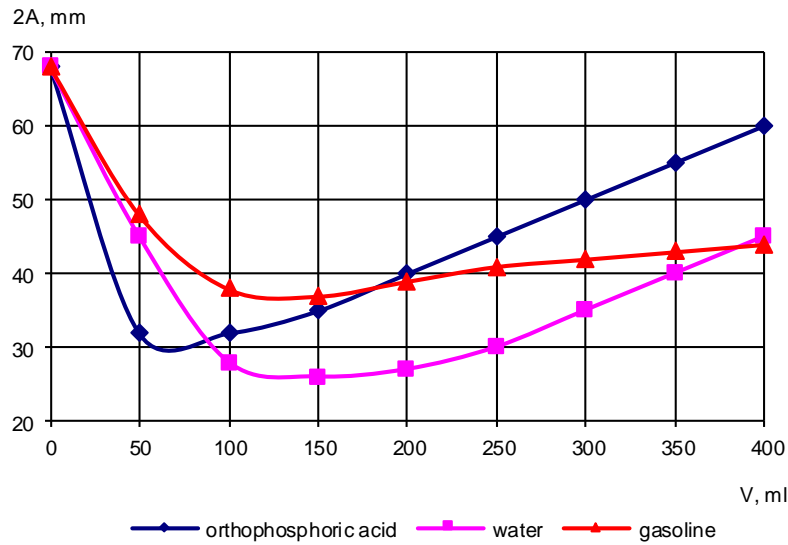

a

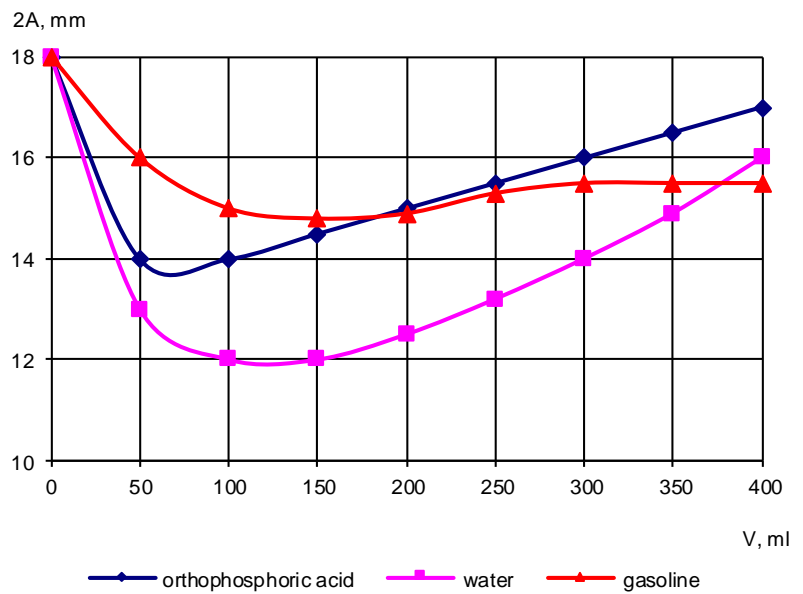

b

Fig. 4 Dependence of the amplitude of oscilations of the upper edge of the cylinder with different filling of the SBD chamber: $a-$ at the resonant frequency, $b-a t$ the operating frequency

Basing on the results of experimental testing (Fig. 4), we can conclude that there is obvious positive effect of automatic balancing with liquid working bodies of different densities for the vertical rotor at rotating at resonance and operating frequencies. The balancing efficiency increases with the approach of the angular velocity to the critical one and with the increase of the phase angle, that is, with the increase of the external resistance. There is an optimal range for the volume of liquid that is installed into the chamber. It ranges from $50 \mathrm{ml}$ to $100 \mathrm{ml}$ for a heavier orthophosphoric acid, 100-150 $\mathrm{ml}$ for fresh water and 150$200 \mathrm{ml}$ for gasoline in the case of an unbalance of $2000 \mathrm{~g}-\mathrm{cm}$. Thus, the optimal volume is proportional to the density of the liquid. In case if the optimum fluid volume in the chamber is reached, further its addition to the chamber leads to the loss of balancing precision. The wave formation on the free surface of the liquid explains this phenomenon. It is known [2] that the zone of instability depends on the mass of the fluid. Therefore, the heaviest liquid - orthophosphoric acid gives the worst efficiency of balancing with the increase of the liquid volume.

Density of the working fluid is insignificant to the process of auto-balancing over the entire range of rotor's frequencies when the SBD chamber is filled with an optimal volume of the liquid. The maximum difference in the amplitudes of oscillations is not more than $10 \mathrm{~mm}$.

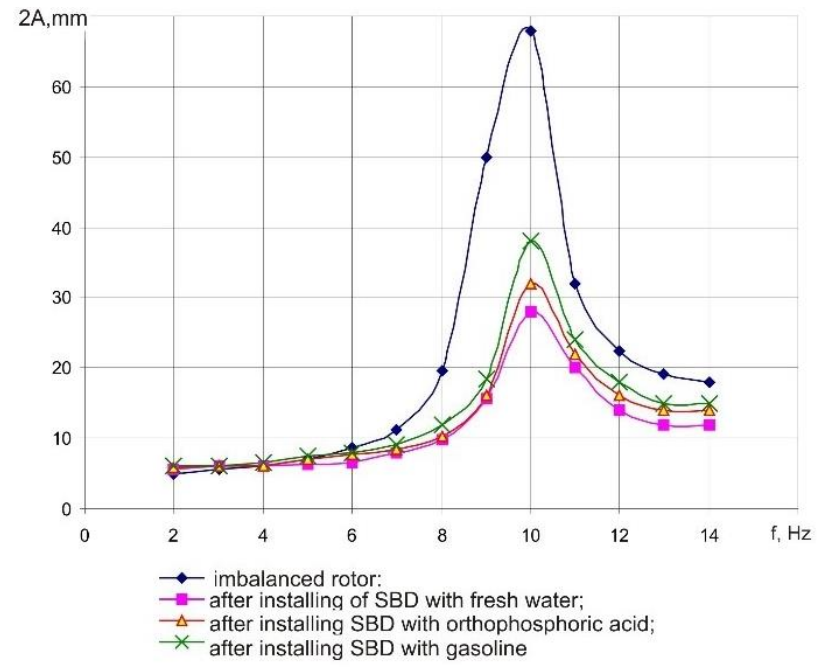

Fig. 5 AFCs of oscillations of the upper edge of the drum at an imbalance of $2000 \mathrm{~g}-\mathrm{cm}$

It is clear from the AFCs of the cylinder with an imbalance of $2000 \mathrm{~g}-\mathrm{cm}$ (Fig. 5) that the proposed liquid SBD allows smooth reaching the working frequencies of rotation. The resonant amplitude of the rotor oscillations decreases by at least $40 \%$ when SBD chamber is filled with a liquid (results presents the case of $100 \mathrm{ml}$ volume of a liquid), and the SBD balances the system even in the resonance zone.

In order to determine the effect of liquid viscosity on the efficiency of the automatic balancing process, a series of experiments with liquids having similar density, but significantly different viscosity have been conducted. Working liquids selected for the experiment: fresh water (density $1000 \mathrm{~kg} / \mathrm{m}^{3}$, kinematic viscosity $1.01 \times 10^{-6} \mathrm{~m}^{2} / \mathrm{s}$ ), sugar syrup No. 1 (density $1230 \mathrm{~kg} / \mathrm{m}^{3}$, kinematic viscosity $14.4 \times 10^{-6} \mathrm{~m}^{2} / \mathrm{s}$, determined experimentally), sugar syrup No. 2 (density $1370 \mathrm{~kg} / \mathrm{m}^{3}$, kinematic viscosity $96.97 \times 10^{-6} \mathrm{~m}^{2} / \mathrm{s}$, determined experimentally). Kinematic viscosity of the used liquids was measured using the Engler viscometer GST6275.

The influence of the viscosity of the balancing fluid on the oscillations of the machine was investigated experimentally by measuring the frequency response of the cylinder oscillations in case of an imbalance of $1000 \mathrm{~g}-\mathrm{cm}$. Measurements were performed on the SBD system without liquid and with the above-mentioned liquids in a $200 \mathrm{~mm}$ radius chamber. Balancing liquids were gradually poured by portions of $50 \mathrm{ml}(50-250 \mathrm{ml})$. The research results are presented in Fig. 6. All curves represent the amplitude of the vibrations of the upper edge of the unbalanced cylinder at constant masses and the position of the imbalance and the different volumes of working fluids. It is evident that liquids of different viscosity but similar density have the same optimal filling volume of 50-100 ml.

Comparison of the corresponding dependencies (Fig. 7), it shows that the viscosity affects oscillations of the rotor significantly. It can be noted that the use of SBD with fresh water leads to a decrease in the double amplitude of the real oscillations (in resonance) from $59 \mathrm{~mm}$ to $24 \mathrm{~mm}$. The use of SBD with syrups leads to a decrease in double amplitude (at resonance) from $59 \mathrm{~mm}$ to $50 \mathrm{~mm}$ for syrup 
No.2 and from $59 \mathrm{~mm}$ to $38 \mathrm{~mm}$ for syrup No.1. Thus, mass forces have less effect on the vertical rotor balancing efficiency than the viscosity of liquid for SBDs.

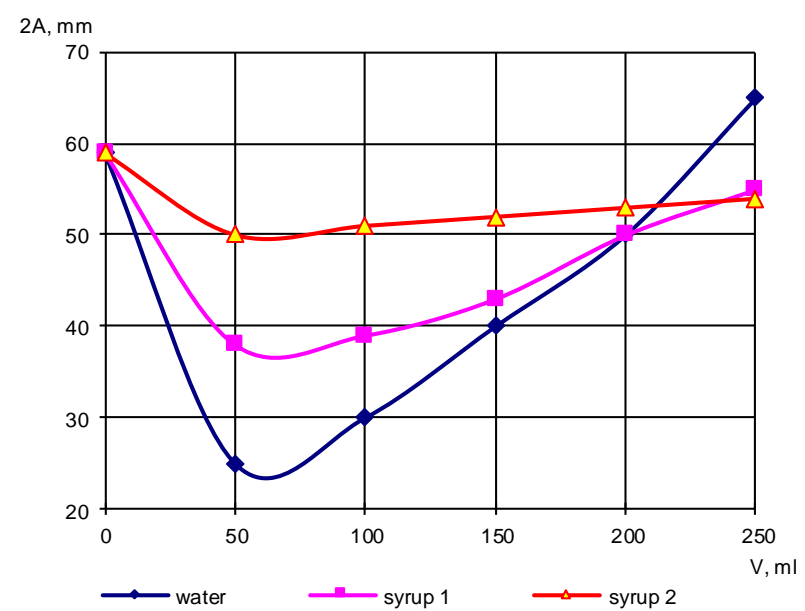

a

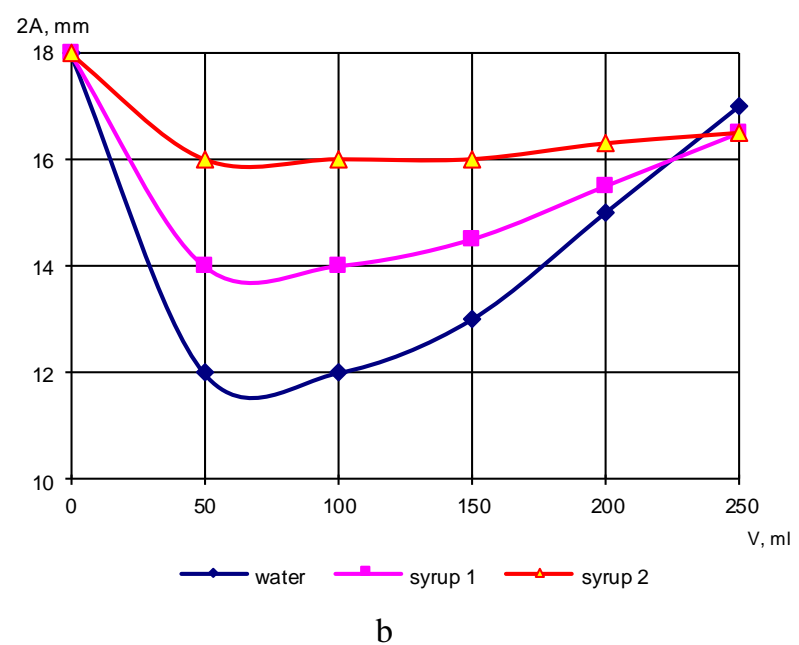

Fig. 6 Dependence of the amplitude of oscillations of the upper edge of the cylinder on the volume of liquid in the SBD chamber: a - at the resonance frequency, $\mathrm{b}-$ at the operating frequency

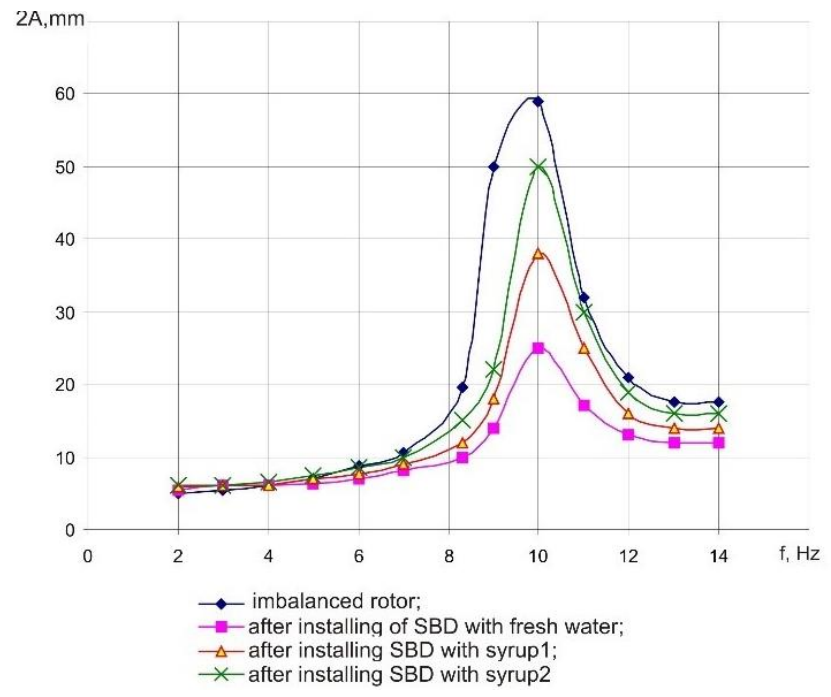

Fig. 7 AFC of oscillations of the upper edge of the drum with an imbalance of $1000 \mathrm{~g}-\mathrm{cm}$

\section{Conclusions}

Results of theoretical and experimental research of auto-balancing devices with liquids lead to the following conclusions:

1. Automatic balancing with the help of liquid working bodies of different densities allows reduction of oscillations amplitudes for the vertical rotor at the pre-resonance, after resonance (operating) and at resonance frequencies.

2. The efficiency of balancing increases with the approaching of the angular velocity to the critical one and with the increase of the phase angle, that is, with the increase of the external resistance.

3. Density of the SBD working (balancing) liquid does not significantly influence the process of self-balancing over the entire frequency range of the rotor rotation.

4. Viscosity of the SBD working (balancing) liquid significantly affects oscillations of the rotor. Consequently, mass forces have less effect on the balancing efficiency than the viscosity for the liquid SBDs.

5. In case the chamber is filled with more liquid than the optimal volume, there is a deterioration in the efficiency of the balancing. That can be explained by the presence of waves on the free surface of a liquid, which causes a dynamic instability in the work of the machine.

\section{References}

1. Royzman, V.; Drach, I., Bubulis, A. 2016. Movement of working fluid in the field of centrifugal forces and forces of weight, In: Proceedings of the 21st International Scientific Conference "Mechanika 12", 222-224.

2. Royzman, V.; Bubulis, A.; Drach, I. 2009. System analysis of automatic balancing (self-balancing) machine totors with liquid working bodies, Solid State Phenomena 147-149: 374-379.

https://doi.org/10.4028/www.scientific.net/SSP.147149.374 .

3. Nikiforov, A. 2013. Condition of the problem of equilibration of rotors, Bulletin of Scientific and Technological Development 4(68): 20-28 (in Russian).

4. Thearle, E. L. 1950. Automatic dynamic balancers (Part 1. Leblanc balancer), Machine Design 22: 119-124.

5. Gusarov, A. A. 2004. Balancing machine rotors. In 2 books. Book 2. M.: Nauka, 266 p.

6. Nesterenko, V.P. 1990. Theory and practice of devices for automatic balancing of rotors: Author's abstract, diss. doctor of technical sciences: 05.02.18 / Novosib. electrical engineering Institute - Novosibirsk, 24-30.

7. Hoon, H. S.; Young, L. J.; Suzuki, S.; Gu, H. W. 2001. A study on the dynamic behaviour of an automatic washing machine, Nippon Kikai Gakkai Kankyo Kogaku Sogo Shinpojiumu Koen Ronbunshu 11: 131-134. https://doi.org/10.1299/jsmeenv.2001.11.131.

8. Pashkov, E.; Martyushev, N.; Ponomarev, A. 2014. Efficiency of balancing by liquid-type automatic balancing devices, Advanced Materials Research: Scientific Journal 1040: 858-863. https://doi.org/10.4028/www.scientific.net/AMR.1040.858. 
9. Pashkov, E.; Martyushev, N.; Masson, A. 2014. Evaluation of gravitational force effect on balancing processes in liquid-type autobalancing devices, Advanced Materials Research: Scientific Journal 1040: 642-649. https://doi.org/10.4028/www.scietific.net/AMR.1040.642.

10. Jung, C. H.; Kim, C. S; Choi, Y. H. J. 2008. A dynamic model and numerical study on the liquid balancer used in an automatic washing machine, Journal of Mechanical Science and Technology 22: 1843-1852. https://doi.org/10.1007/s12206-008-0623-2.

11. Langthjem, M. A.; Nakamura, T. 2013. Dynamics of the fluid balancer: Perturbation solution of a forced Korteweg-de Vries-Burgers equation. RIMS, Kyoto University 1847: 73-85.

12. Cunico, M. W. M. 2015. Characterization and modelling of LeBlanc Hydrodynamic Stabilizer: a novel approach for steady and transient state models, Modelling and Simulation in Engineering 2015: 11.

http://dx.doi.org/10.1155/2015/729582.

13. Chen, H. W.; Zhang, Q.; Fan, S. Y. 2011. Study on steady-state response of a vertical axis automatic washing machine with a hydraulic balancer using a new approach and a method for getting a smaller deflection angle, J. Sound Vib. 330: 2017-2030. https://doi.org/10.1016/j.jsv.2010.11.006.

14. Kim, K.; Kim, J.; Park, S. 2017. Laundry treating apparatus: patent US 9663889 B2 / Owner name: Lg Electronics Inc. [Online], [accessed 30 May 2019]. Available from Internet:

http://www.google.com/patents/US9663889 (may 30, 2017).

15. Kim, K.; Park, S.; Kim, J. 2016. Balancing unit and laundry treatment apparatus EP 3085827 A1 / Owner name: Lg Electronics Inc. [Online], [accessed 16 May 2019]. Available from Internet: http://www.google.com/patents/EP3085827A1?cl=en.

16. Ostdiek, S. D.; Verma, V. 2012. Laundry treating appliance with balancing system: patent US 20120144598 A1 / Owner name: Whirlpool Corporation. [Online], [accessed 24 May 2019]. Available from Internet: http://www.google.com/patents/US20120144598.

17. SKF- Automatic balancing units (2017) Automatic balancing units. [Online], [accessed 6 June 2019]. Available from Internet: http://www.skf.com/us/industry-solutions/portable-power-tools/applications/grinders-planners-and-sanders/automatic-balancing-unit/index.html.

18. Kim, Y. K.; Lee, K. C.; Ko, H. S.; Lee, S. U. 2015 Washing machine, inner tube of washing machine and balancer connection and assembly method thereof: pa- tent US 8984918 B2 / Owner name: Samsung Electronics Co., Ltd. [Online], [accessed 8 April 2019]. Available from Internet:

https://patents.google.com/patent/US8984918.

19. Royzman, V.; Drach, I.; Tkachuk, V.; Pilkauskas, K.; Čižauskas, G.; Šulginas, A. 2018. Operation of passive fluid self-balancing device at resonance transition regime, Mechanika 24(6): 805-810. https://doi.org/10.5755/j01.mech.24.6.22469.

20. Altshul, A. D.; Kiselev, P. G. 1975. Hydraulics and aerodynamics (Fundamentals of fluid mechanics). A manual for high schools: Moscow, Stroyizdat 24-25.

I. Drach, V. Royzman, A. Bubulis, K. Juzėnas

\section{PASSIVE BALANCING OF THE ROTOR WITH AN AUTO-BALANCING DEVICE WITH A VISCOUS INCOMPRESSIBLE LIQUID}

S u m m a r y

The use of liquid auto-balancers to compensate the operational changes in the imbalance of rotary systems without stopping them is of interest because of the relative structural simplicity of these devices, which are passive direct-acting regulators that do not require power supply and control systems to move correction masses. The experience of the study of passive auto-balancing devices (SBD) indicates that the existing theory (statements) of passive automatic balancing of the fluid is idealized and inaccurately describes the processes that occur with the working bodies during their operation. In particular, the lack of studies on the effect of liquid viscosity on the efficiency of self-balancing and the reasonableness of the selection of liquid during the development of fluid SBD demanded to analyse the operation of liquid SBD in the real system, taking into account the influence of liquid properties on the efficiency of the vertical rotor balancing process. It is shown that the efficiency of balancing increases with the approach of the angular velocity to the critical one and with the increase of the external resistance. The massive forces of the working fluid have less effect on the balancing efficiency than the viscosity for liquid SBDs.

Keywords: rotary system, oscillations, imbalance, balancing efficiency, auto-balancing device, viscosity.

Received July 08, 2019

Accepted February 17, 2021 